\title{
Let's Come Together - Social Navigation Behaviors of Virtual and Real Humans
}

\author{
Matthias Rehm, Elisabeth André, and Michael Nischt \\ Augsburg University, Institute of Computer Science, \\ 86136 Augsburg, Germany \\ \{rehm, andre\}@informatik.uni-augsburg.de \\ http://mm-werkstatt.informatik. uni-augsburg.de
}

\begin{abstract}
In this paper, we present a game-like scenario that is based on a model of social group dynamics inspired by theories from the social sciences. The model is augmented by a model of proxemics that simulates the role of distance and spatial orientation in human-human communication. By means of proxemics, a group of human participants may signal other humans whether they welcome new group members to join or not. In this paper, we describe the results of an experiment we conducted to shed light on the question of how humans respond to such cues when shown by virtual humans.
\end{abstract}

\section{Introduction}

Synthetic agents have been employed in many games and entertainment applications with the aim to engage users and enhance their experience. However, to achieve this goal, it does not suffice to provide for sophisticated animation and rendering techniques. Rather, other qualities have to come in focus as well, including the provision of conversational skills as well as the simulation of social competence that manifests itself in a number of different abilities.

While earlier work focused on one specific aspect of social behavior, such as the expression of socially desirable emotions, more recent research aims at the operationalization of complex models of social behavior between members of a group including emotions, personality and social roles as well as their dynamics. For instance, Prendinger and Ishizuka [16] investigate the relationship between an agent's social role and the associated constraints on emotion expression. They allow a human script writer to specify the social distance and social power relationships among the characters involved in an application, such as a multi-player game scenario. Another approach has been taken by Rist and Schmitt [19] who aim at emulating dynamic group phenomena in human-human negotiation dialogues based on sociopsychological theories of cognitive consistency dynamics [13]. To this end, they consider a character's attitudes towards other characters and model a character's social embedding in terms of liking relationships between the character and all other interaction partners. Prada and Paiva [15] as well as Marsella and colleagues [17] developed a social simulation tool as a backend to interactive pedagogical drama applications. While the development of social 
relationships in the approach by Prada and Paiva [15] is mainly determined by the type of social interactions between them, Marsella and colleagues regard the beliefs of agents about other agents as a key factor of social interaction and rely on a theory of mind to explicitly represent the beliefs of agents about other agents. Commercial games, such as TheSims, show that the simulation of social skills may render interactions between virtual characters more believable and engaging.

In the systems described above, social behaviors are mainly reflected by the agents' communicative behaviors. In contrast, Thalmann and colleagues [5] concentrate on the simulation of social navigation behaviours in virtual 3D environments including the social avoidance of collisions, intelligent approach behaviours, and the calculation of suitable interaction distances and angles. The work is based on an operationalization of empirically-grounded theories of human group dynamics, such as Kendon's group formation system [9].

The objective of our work is to investigate navigation behaviors of humans that socially interact with virtual agents. Unlike Thalmann and colleagues [5] who focus on the simulation of human navigation behaviors using virtual agents, we involve the users as active participants in the scenario. In particular, we allow them to freely navigate through the scenario and join or leave groups of other agents by making use of a commercially available dancing pad that is employed in many computer games. As a consequence, interaction requires the users to move their full body in the physical space.

Our approach is closely related to projects that make use of proxemics as an interaction parameter. An example of a proximity-based game includes Pirates [4]. In this game, possible actions are triggered if the player is close to a certain location. For instance, to attack another player, one has to be physically close to the other player, otherwise this option is not available. Another proximity-based game is MirrorSpace by Roussel and colleagues where the display of information is affected by the distance of the user to the screen [20]. Partala and colleagues studied the influence of an agent's proximity on the affective response of the user [14]. Whereas valence and arousal seemed not to be influenced by proximity variances, a significant effect was found for the dominance dimension.

In the next section, we first present a model for group dynamics that simulates changes in the social relationships between agents as a side effect of social interactions. Based on the model, we designed and implemented a game-like scenario which has been employed as a test bed to study social navigation behaviors of humans interacting with virtual agents. The model is augmented by a model of proxemics that simulates the role of distance and spatial orientation in human-human communication. By means of proxemics, a group of human participants may signal other humans whether they welcome new group members to join or not. The paper will report on the outcome of an experiment we conducted to investigate how humans respond to such cues when they are elicited by virtual agents. In particular, we were interested in the question of whether humans prefer to join open group formations as opposed to closed group formations. 


\section{Description of the Underlying Model}

Inspired by work on social psychological theories of group behaviors, we have designed and implemented a computational model of group dynamics.

\subsection{Representation of Interpersonal Relationships}

The profile of the single agents is characterized by their name, gender, marital status, age group, social status, sex orientation and personality. An agent's personality is represented by a vector of discrete values along a number of psychological traits, such as extraversion or agreeableness, that uniquely characterize an individual [11].

Furthermore, the model is based on an explicit representation of the relationship between single agents, the relationship between agents and the groups they belong to and the attitude of agents towards objects. Interpersonal relationships are described by the degree of liking, familiarity, trust and commitment. These values are either specified by the user in advance or derived from known properties of the agent's profile. For instance, agents with a similar social status are considered to trust each other more than agents with a different social status. The social role of an agent within a group is described by features, such as its power, prestige and affiliation.

\subsection{Development of Interpersonal Relationships}

To model how social interactions influence the social relationships between agents, we start from Interaction Process Analysis (IPA) [2]. IPA is based on a classification of social interactions that take place in small groups and essentially distinguishes between socio-emotional factors that refer to the social relationships within a group, such as positive or negative feedback to group members, and task-oriented factors that refer to group tasks, such as asking questions or summarizing and offering direction. It has already been successfully employed in other system of social group dynamics, see for example [5] or [15].

To determine the type and number of social interactions, we use ideas from social impact theory [8] which defines how the presence of others influences one's behavior. The strength of this influence is calculated in a close analogy to physical phenomena, such as the amount of light visible on a table which depends on the number of light sources, their distances to the table and their strength. The social impact on a target person is calculated taking into account the strength, immediacy, and number of source persons where strength comprises features, such as status or power, and immediacy represents the physical distance between source and target. As any of these factors increases, the impact on the target also increases. For instance, if a subject has to perform a song, stage fright increases with the number of people in the audience and their status. It decreases if the subject has not to perform alone [7].

Furthermore, we were inspired by self attention theory [12]. Self attention theory is a theory of self-regulation that explains behavior modifications if one 


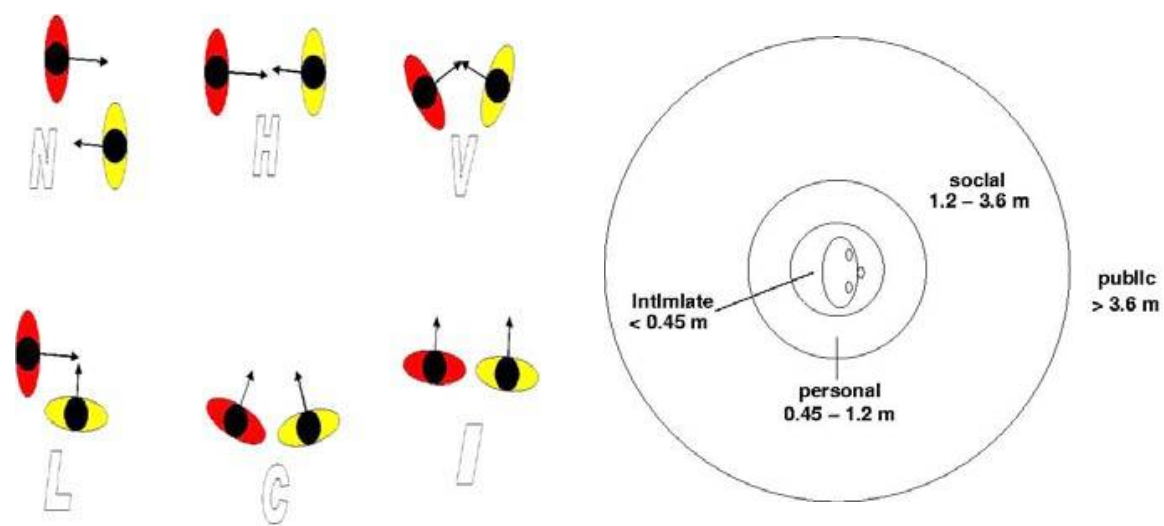

Fig. 1. Orientation variants of two agents (left) and distance zones for an agent (right)

is the subject of one's own attentional focus. In this case, violations of standard social norms will be more salient. People's peer groups influence self attention and regulation in at least two different ways. Behavioral standards will be set by the group to which the individual has to adhere on the one hand. On the other hand, group size matters. Larger groups result in decreased self awareness because single individuals will easier go unnoticed. The so called other-total ratio is used to describe this effect for the interaction between arbitrary groups. It represents the proportion of the total group that is comprised of people in the other subgroup.

The influence between the social configuration of a group and the number and type of interactions is bi-directional. To describe how the nature of the interactions influences the development of interpersonal relationships between group members, we follow ideas by Schmitt [21] and make use of the "Congruity Theory" by Osgood and Tannenbaum [13]. The theory is based on the hypothesis that people tend to avoid unbalanced configurations or cognitive dissonances. For instance, when a statement of a speaker causes such an unwanted state in the addressees, they will either change their attitudes towards the subject matter, or their attitudes towards the speaker who caused a dissonance. As a consequence, the theory allows us to describe changes in social relationships as a side effect of interactions between agents.

\subsection{Social Navigation}

As noted earlier, we make use of proxemics as an interaction parameter. According to Hall, proxemics is the investigation of man's spatial behavior which is of importance because "space is one of the basic, underlying organizational systems for all living things - particularly for people" [6].

One determinant of such spatial behavior is the acceptable distance between communication partners which influences their spatial interaction behavior. Hall 

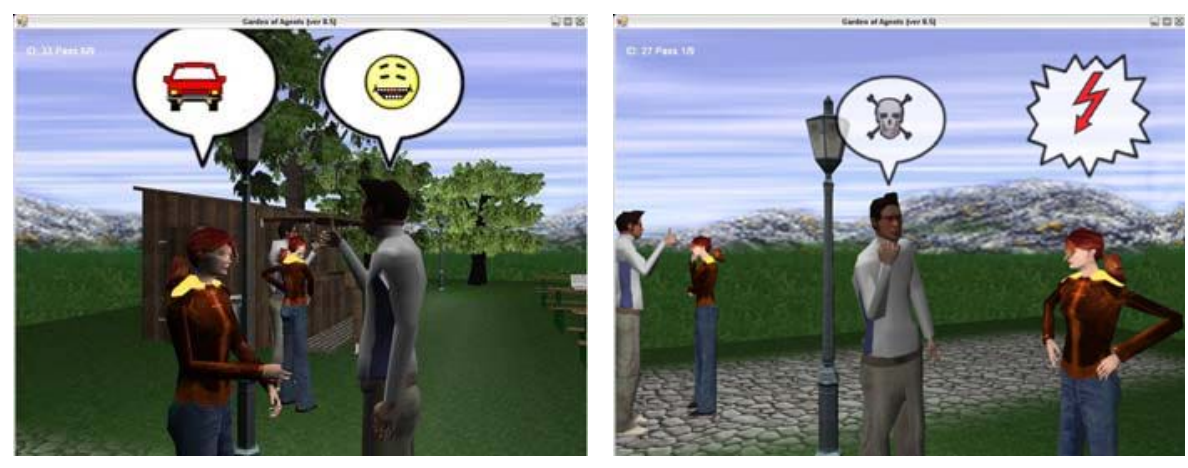

Fig. 2. Interacting agents

[6] distinguishes four different distances that are related to behavior changes which occur if someone enters these distance zones. Intimate distance ranges up to $45 \mathrm{~cm}$ and is reserved for very close relationships. Personal distance ranges from $45 \mathrm{~cm}$ to $1.2 \mathrm{~m}$ which allows touching and is thus reserved for focused and private interactions. Social distance ranges from $1.2 \mathrm{~m}$ to $3.6 \mathrm{~m}$, public distance starts at $3.6 \mathrm{~m}$. The specific distances given by Hall are valid for white Northern Americans. Whereas the existence of the four different distances seems to be universal, the ranges itself and acceptable behaviors related to the distance zones are culture specific. Relying on Hall, Knowles [10] has developed a straightforward model based on proximity to handle the effects of crowding. In essence, the influence of others results from a combination of their number and their distance from the target person.

Whereas Hall's analysis is primarily concerned with distances, Kendon takes also the orientation of the interlocutors into account. Based on [9], a model for the interaction between pairs of people was integrated. Depending on their interpersonal relations, people will orient themselves differently when joining others in public places. The six most frequent orientations for communicating pairs were taken into account (see Fig. 1 left). Half of these are so called closed (upper line), the other half open orientations (lower line). Closed orientations indicate that the interlocutors won't be disturbed by other people, whereas interloctors in open orientations will allow others to enter the conversation.

\section{The Beergarden Scenario}

To test our model, we have decided to implement a virtual beergarden where agents wander around to meet friends or to build up new relationships. The initial position of the agents' in the scenario is randomly assigned by the system. When the system is started, the agents perform a random walk until they perceive another agent or a group they wish to start a conversation with. 
Figure 2 shows two different pairs of agents interacting with each other. On the left hand side a task-oriented interaction is depicted. The female agent brought up the topic of a car which is indicated by the car icon. The male agent gives his positive opinion on this topic indicated by the icon as well as his gesture (thumbs up). The pair on the right of Figure 2 finds itself in a negative social emotional interaction where the male agent shows antagonism indicated by the skull icon and his rude gesture (showing a fist). The female agent shows tension by "shouting" (indicated by the jagged outline of her speaking bubble), producing the lightning icon and putting her hands on her hips.

The agents' behavior is determined by a de-centralized behavior control mechanism that relies on rules derived from the theories described in Section 2. The left-hand side of a rule specifies a condition that has to be fulfilled to apply a rule, the right-hand side either refers to an elementary action or a complex behavior script that indicates what a character should do in a certain situation. For instance, we have defined several greeting scripts consisting of distance salutation, approach and close salutation based on [9]. To elicit a greeting script, a character must sight another character, identify it as someone it wishes to greet and believe that the other character is aware of it. At runtime, scripts are decomposed into elementary actions that are forwarded to the animation engine. For each elementary action, we have modeled a set of specific animation sequences. For the IPA actions, we modeled a number of postures and gestures relying on the descriptions in the Berlin dictionary of everyday gestures ("Berliner Lexikon der Alltagsgesten", [3]).

The current animation engine is built upon Managed DirectX to efficiently render the visual content of a scene and access the programmable pipeline stages of modern GPUs. As the individual characters lie in the main focus, their geometry can be modified using a variety of techniques, including skeletal-subspacedeformation (a.k.a. vertex skinning) and morph targets. This allows us to simulate body motions and gestures based on forward/inverse kinematics as well as to represent mimics by state vectors, which easily can be interpolated. Moreover, a fast and configurable path finding routine tells the virtual agents how to reach a certain place while distances to the others are maintained depending on their social relationships. In order to clearly separate the agents' animation engine from the underlying behavior control mechanism, all types of animations can be triggered from an application-independent interface, which comprises a perception model, encapsulating qualitative information about spatial relations based on the position and orientation of the agent.

\subsection{User Study}

The Beergarden allows us to perform controlled experiments to shed light on the question of how users respond to encounters with agents that follow the aforementioned social navigation behaviors. Here, we will report on an experiment we conducted to find out whether users feel more encouraged to join open group formations as opposed to closed group formations. Furthermore, we investigate what social distance they maintain when deciding to join a group. 


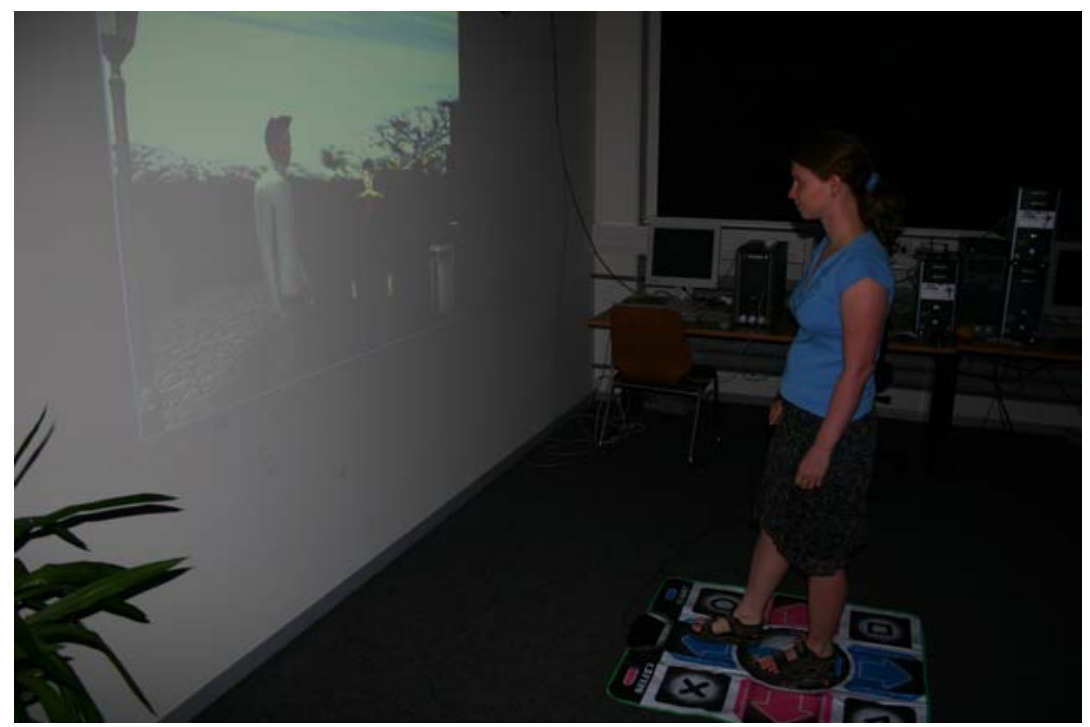

Fig. 3. Joining a group of agents

According to the media equation [18], it should not make a great difference whether humans approach a virtual agent or another human. Indeed a number of studies by Bailenson and colleagues [1] revealed that the size and the shape of the personal space around virtual humans resembled the shape of the space that people leave around real, nonintimate humans. Nevertheless, Bailenson and colleagues also observed that the social navigation behavior depends on whether the agent is controlled by a computer or by a real human. In particular, they found out that people gave an avatar more personal space than an autonomous agent. In a second experiment, they examined what happens when virtual humans walk and violate the personal space of the participants. The experiment showed that human subjects avoided the virtual human more when it was obviously computer-controlled than an avatar that was controlled by another human.

To investigate these questions, the following experimental setting was set up (see Fig. 3). The user was enabled to navigate by means of a pressure-sensitive dancing pad and with a first person view through the beergarden. The choice of the dancing pad instead of e.g., a joy pad or the keyboard, made sure that subjects were involved with their full body in the experience of navigating through the beergarden. Because the general scenario described above would have been to unconstrained, the number of agents was restricted to two groups with two agents each. The subjects were instructed to join one of the groups present in the beergarden. The graphical icons denoting the communicative meaning were disabled to prevent users from choosing a group on the basis of the communicative content. Each scenario consisted of two groups of agents where one group was positioned in an open $(\mathrm{L}, \mathrm{C}, \mathrm{I})$, the other in a closed form $(\mathrm{H}, \mathrm{N}, \mathrm{V})$. The nine 
Table 1. Orientation of agents in groups joined by subjects

\begin{tabular}{|ccc|ccc|}
\hline Conf. $\#$ & $\%$ & Class & $\#$ & $\%$ \\
\hline \hline $\mathrm{H}$ & 11 & $5.1 \%$ & & & \\
$\mathrm{~N}$ & 3 & $1.4 \%$ & closed 34 & $16 \%$ \\
$\mathrm{~V}$ & 20 & $9.3 \%$ & & & \\
\hline $\mathrm{C}$ & 52 & $24.1 \%$ & & & \\
L & 72 & $33.3 \%$ & open & 182 & $84 \%$ \\
$\mathrm{I}$ & 58 & $26.9 \%$ & & & \\
\hline
\end{tabular}

Table 2. Distance of subjects to agents

\begin{tabular}{|c|c|c|c|c|}
\hline Distance & intimate & personal & $\begin{array}{c}\text { social } \\
\text { close far }\end{array}$ & public \\
\hline \# & 0 & 0 & $\begin{array}{|ll|}7 & 5\end{array}$ & 0 \\
\hline
\end{tabular}

resulting combinations were tested twice with each subject, changing the left and right position of the groups. To control for gender differences, each group was composed of one female and one male agent. For each subject, the 18 scenarios were presented in random order.

10 students and 2 staff members from the computer science department participated in this experiment ranging in age from 22 to 35 . Nine subjects were male and three female. Ten of the subjects had a German, two an Arabic cultural background. This is of interest because studies in cross-cultural communication (e.g., see [22] and [6]) show that the proxemic behavior of these two cultures is noticeably different. Subjects were instructed to join one of the groups in each scenario. Prior to the experimental session, the subjects had the opportunity to familiarize themselves with the dancing pad and the beergarden environment which they could freely explore as long as they liked.

Results. In $84 \%$ of the scenarios, the subjects joined the group which was positioned in an open formation. Two of the subjects never navigated to one of the other formations, another two chose closed formations in a third of the time (see Table 1). A paired-samples t-test showed that this difference is significant for $p<0.01$. In 20 from the remaining 34 scenarios in which a closed formation was chosen, it was the $\mathrm{V}$-formation which can sometimes be mistaken for a less open C-formation.

The result for the proxemic behavior of the subjects is non-ambiguous (see Table 2). For all scenarios, subjects positioned themselves in a social distance which has a range between $1.2 \mathrm{~m}$ to $3.6 \mathrm{~m}$ with a close area from $1.2 \mathrm{~m}$ to 2.1 $\mathrm{m}$ and a far area ranging from $2.1 \mathrm{~m}$ to $3.6 \mathrm{~m}$. From the German subjects, five positioned themselves in the far area and five in the close area. In general, such a behavior also depends on the personality of people [6] and is influenced by their cultural background. We did not test the personality influence in this study. With respect to the cultural background parameter, we observed the following. 
The two Arabic subjects positioned themselves in the close area. With only two subjects with this cultural background, not very much can be said about this effect - only that they positioned themselves as predicted. One of the subjects was well aware of the cultural differences in proxemic behavior because during the experiment he explained that he does not move closer because he didn't want to terrify the guys (which he considered to have a different cultural background due to their appearance).

\section{Conclusions}

In this paper we described a multi agent system in which the development of interpersonal relationships as well as the control of interactions is based on models of social group behavior. To integrate the user in this system and allow her to freely interact with the agents we devised a first experiment to shed light on the question of how the proxemic behavior of users in this scenario adheres to results found in the literature. By navigating through the environment employing a dancing pad subjects experienced full body movements. So far, the reciprocal interactions between the virtual humans and the humans are still very limited since the model described in Section 2 was mainly used to simulate the social group dynamics of virtual humans (as opposed to virtual AND real humans). Nevertheless, the positioning behavior of the human subjects can be described as natural. They joined open group formations of agents significantly more often than closed ones and positioned themselves in a social distance. Thus, the first step of integrating the user was successful.

During the experiment, some of the male subjects expressed their preferences for the female agents. All groups were composed of one male and one female agent to control for such possible gender differences. Next, the users will be confronted with purely male and female groups to test for this difference which might well override a choice based solely on open vs. closed formations. We will also look more closely at the interaction angles of the humans which we recorded in the experiment, but which were not yet analyzed. Finally, we will investigate the influence of non-verbal agent behaviors, such as looking at the users.

A different line of research will concentrate on modelling more sophisticated proxemic behavior in agents. Hall [6] gives some insights in the relationship between distance and non-verbal behavior which changes according to the distance between interlocutors. For instance, the farther away interlocutors are from each other, the louder they speak, starting from whispering in an intimate distance to shouting in a public distance.

\section{References}

1. J. N. Bailenson, J. Blasovich, A. C. Beall, and J. M. Loomis. Interpersonal distance in immersive virtual environments. Personality and Social Psychology Bulletin, pages 819-833, 2003.

2. R. F. Bales. Interaction Process Analysis. Chicago University Press, Chicago, 1951. 
3. BLAG. Berliner lexikon der alltagsgesten. http://www.ims.uni-stuttgart.de/ projekte/nite/BLAG/, last visited: 22.03.2005.

4. Jennica Falk, Peter Ljungstrand, Staffan Björk, and Rebecca Hansson. Pirates: Proximity-Triggered Interaction in a Multiplayer Game. In Proceedings of CHI, pages 119-120, 2001.

5. A. Guye-Vuillième and D. Thalmann. A high level architecture for believable social agents. Virtual Reality Journal, 5:95-106, 2001.

6. Edward T. Hall. The Hidden Dimension. Doubleday, 1966.

7. J. M. Jackson and B. Latané. All alone in front of those people: Stagefright as a function of number and type of coperformers and audience. Journal of Personality and Social Psychology, (40):72-85, 1981.

8. Jeffrey M. Jackson. Social Impact Theory: A Social Forces Model of Influence. In Brian Mullen and George R. Goethals, editors, Theories of Group behavior, pages 111-124. Springer, New York, Berlin, 1987.

9. Adam Kendon. Conducting Interaction: Patterns of Behavior in Focused Encounters. Cambridge Univ Press, Cambridge, 1991.

10. E. S. Knowles. Social physics and the effects of others: Tests of the effects of audience size and distance on social judgements and behavior. Journal of Personaliy and Social Psychology, (45):1263-1279, 1983.

11. R. R. McCrae and O. P. John. An introduction to the five factor model and its applications. Journal of Personality, (60):175-215, 1992.

12. Brian Mullen. Self-Attention Theory: The Effects of Group Composition on the Individual. In Brian Mullen and George R. Goethals, editors, Theories of Group behavior, pages 125-146. Springer, New York, Berlin, 1987.

13. C. E. Osgood and P. H. Tannenbaum. The Principle of Congruity in the Prediction of Attitude Change. Psychological Review, (62):42-55, 1955.

14. Timo Partala, Veikko Surakka, and Jussi Lahti. Affective Effects of Agent Proximity in Conversational Systems. In Proceedings of NordCHI, pages 353-356, 2004.

15. Rui Prada and Ana Paiva. Intelligent virtual agents in collaborative scenarios. In Proceedings of Intelligent Virtual Agents (IVA), pages 317-328, 2005.

16. Helmut Prendinger and Mitsuru Ishizuka. Social Role Awareness in Animated Agents. In Proceedings of Agents '01, Montreal, Canada, pages 270-277, 2001.

17. D. V. Pynadath and S. C. Marsella. PsychSim: Modeling Theory of Mind with Decision-Theoretic AgentsM. In Proceedings of the Fifteenth IJCAI. Morgan Kaufman Publishers Inc., 2005. to appear.

18. Byron Reeves and Clifford Nass. The Media Equation - How People Treat Computers, Television, and New Media Like Real People and Places. Cambridge University Press, Cambridge, 1996.

19. Thomas Rist and Markus Schmitt. Applying socio-psychological concepts of cognitive consistency to negotiation dialog scenarios with embodied conversational characters. In AISB Symposium on Animated Expressive Characters for Social Interactions, 2002.

20. Nicolas Roussel, Helen Evans, and Heiko Hansen. Utilisation de la distance comme interface à un système de communication vidéo. In Proceedings of IHM, pages 268 271. ACM, 2003.

21. Markus Schmitt. Dynamische Modellierung interpersoneller Beziehungen zwischen virtuellen Charakteren. PhD thesis, Universität des Saarlandes, Saarbrücken, 2005.

22. Stella Ting-Toomey. Communicating Across Cultures. The Guilford Press, New York, 1999. 